\title{
Ways of intensification of the process of moistening the grain before grinding
}

\author{
Lyudmila V. Larina, Dmitry V. Rusljakov*, Tatiana I. Tupolski, and Olga B. Tikhonova
}

Don State Technical University, 344002 Gagarin Square, 1, Rostov-on-Don, Russian Federation

\begin{abstract}
The aim of this study is substantiation of ways of reducing the time required to process pre-wetting the grain before peeling, flattening, grinding [1]. A method of moistening the grain before grinding with preevacuation of its interior microvoids through the porous shell provides moisture transfer in the liquid phase. This moisture transfer requires the subsequent application of excess pressure to the penetration of moisture to the core of the grain and subsequent long-term otvalivayas grain. To significantly reduce the time of wetting is proposed to use a moisture transfer with effusion in the form of steam produced at low pressure (vacuum), then the guaranteed condensation in micropolitan porous shell of the grain. Study of intensity of moisture transport in accordance with the changes in concentrations of vapour along the length of the capillary in a vacuum proved the possibility of intensifying the process of moistening the grain before grinding.
\end{abstract}

\section{Introduction}

A large number of agricultural processes includes the operations of wetting, which contribute to the plasticization of the grain, reducing the time of fragmentation with its further peeling, to improve the quality of the finished product. Reduction of time consumption involves the use for this purpose of the method of moistening the grain before grinding [2]. This method includes the following operations: 1) pre-evacuation internal microvoids grain through its porous shell; 2) moisture by spraying with water; 3 ) application of excess pressure, contributing to the forced entry of surface moisture into the vacuumed grain micropoles; 4) the courage of the grain. In the capillary-porous shell of grain, moisture transfer can be carried out in vapor (effusion), liquid (diffusion) States, in the form of a film flow. Consideration of the contribution of each of these processes to the total moisture transfer will allow us to provide the intensification of the process of wetting using a vacuum [3].

\section{Basic formulas for different methods of moisture transfer}

For the phase estimation of the moisture transfer through the pre-vakuumirovannoj porous shell in the inner cavity of the grain using the first law of Fick [4]:

\footnotetext{
* Corresponding author: ruslyakof@yandex.ru
} 


$$
\Delta M=-D \frac{d \rho}{d x} \cdot \Delta S \cdot \Delta \tau
$$

where $\Delta M$ - the mass of moisture carried over time $\Delta \tau$ through the elementary area $\Delta S$ in the direction of the normal $\mathrm{x}$ to the site in the direction of decreasing density; $\frac{d \rho}{d x}-$ density gradient; $D$ - diffusion coefficient.

The diffusion coefficient in a gas medium according to the elementary kinetic theory of gases is determined by the formula [5]:

$$
D=\frac{1}{3} \bar{u} \cdot \bar{\lambda}
$$

where $\bar{u}$ - the average speed of thermal motion of molecules; $\bar{\lambda}-$ the average length of free path of molecules.

The mean free path length of molecules is determined by the formula [5]:

$$
\bar{\lambda}=\frac{k T}{\pi \sqrt{2 \cdot} d^{2} \cdot p},
$$

where $k=1,38 \cdot 10^{-23} \mathrm{joul} / \mathrm{K}$ is the Boltzmann's constant; $T$ - temperature in Kelvin; $d$ - is the diameter of the molecule; $p$ - is the gas pressure.

At $T=323 \mathrm{~K} / 50^{\circ} \mathrm{C}$ and the corresponding the equilibrium vapor pressure of water $p=12,3 \cdot 10^{3}$. The mean free path length of the water molecule with a diameter $3,0 \cdot 10^{-10}$ of $m$ will be:

$$
\bar{\lambda}=\frac{1,38 \cdot 10^{-23} \cdot 323}{3,14 \sqrt{2} \cdot\left(3 \cdot 10^{-10}\right)^{2} \cdot 12,3 \cdot 10^{3}}=0,906 \cdot 10^{-6} \mathrm{M} .
$$

Average speed of thermal motion of water molecules, we define by the formula [5]:

$$
\bar{u}=\sqrt{\frac{8 R T}{\pi \cdot \mu}} \approx 1,60 \sqrt{\frac{k T}{m}},
$$

where $m$ - is the mass of a water molecule equal to $29,9 \cdot 10^{-27} \mathrm{~kg}$.

$$
\bar{u}=1,60 \sqrt{\frac{1,38 \cdot 10^{-23} \cdot 323}{29,9 \cdot 10^{-27}}} \approx 6,17 \cdot 10^{2} \mathrm{M} / \mathrm{c}
$$

The diffusion coefficient of steam according to the formula (2) will be:

$$
D=\frac{1}{3} \cdot 6,17 \cdot 10^{2} \cdot 0,906 \cdot 10^{-6}=1,87 \cdot 10^{-4} \mathrm{M}^{2} / \mathrm{c}
$$

The diffusion coefficient of liquid moisture is determined by the formula:

$$
D_{1}=\frac{k T}{6 \pi \cdot \eta \cdot d}
$$

where $\eta_{0}-$ is the dynamic coefficient of viscosity of water.

For its calculation use the formula of Poiseuille:

$$
\eta=\eta_{\mathrm{o}}\left[1+0,0377 \cdot(T-273 K)+0,000221 \cdot(T-273 K)^{2}\right]^{-1},
$$

where $\eta_{\mathrm{o}}-$ is the value of the dynamic viscosity coefficient (at $T=273 K$, $\left.\eta_{\mathrm{o}}=17,9 \cdot 10^{-4} \mathrm{Ns} / \mathrm{m}\right)$.

Then at $T=323 \mathrm{~K}$ we have: 


$$
\eta=17,9 \cdot 10^{-4}\left[1+0,0377 \cdot 50+0,000221 \cdot 50^{2}\right]^{-1}=5,21 \cdot 10^{-3} \mathrm{Ns} / \mathrm{m}^{2} .
$$

The diffusion coefficient of liquid moisture $D_{l}$ will be:

$$
D_{1}=\frac{1,38 \cdot 10^{-23} \cdot 323}{6 \cdot 3,14 \cdot 5,21 \cdot 10^{-3} \cdot 3,0 \cdot 10^{-10}}=1,51 \cdot 10^{-10} \mathrm{~m}^{2} / \mathrm{s}
$$

As follows from the obtained calculations, the comparison of the transport intensity of liquid moisture, characterized by a diffusion coefficient $D_{I}=1,87 \cdot 10^{-4} \mathrm{~m}^{2} / \mathrm{s}$ with the transport intensity of vapor moisture, characterized by a coefficient $D=1,51 \cdot 10^{-10} \mathrm{~m}^{2} / \mathrm{s}$, indicates a significant degree of intensification of the moisture transfer process in the vapor state (more than six orders of magnitude higher) than in the liquid.

Thus, when using the preliminary vacuuming of internal micro-cavities of grain through its porous shell to reduce the time spent on humidifying the grain before grinding, it is necessary to take into account the moisture transfer through the capillaries (pores) of the grain shell in the gaseous phase as more effective.

\section{Research part}

Investigation of the intensity of steam transport when its concentration changes along the length of the pre-vacuumed capillary. Physical model of the process: 1) capillary-cylindrical tube volume $-V_{\text {тр. }}$, long- $1_{\text {тр. }}$, diameter- $\mathrm{d}_{\text {тр. }}$. with flap between one of the tube bases and the capacity of the volume $-\mathrm{V}_{\text {ем. }}$. with water vapor; 2) $\mathrm{V}_{\text {ем. }}>>\mathrm{V}_{\text {тр. }}$; 3) $1_{\text {тр. }}>>\mathrm{d}_{\mathrm{tp}}$. Under these conditions and the removed flap at the time $\tau>0$, the task of investigating the intensity of steam transport is one-dimensional.

The origin is located on the border between the tube and the water vapor tank. The $x$ axis is directed into the tube.

The diffusion equation in this case looks like [6]:

$$
\frac{d u}{d \tau}=D \frac{d^{2} u}{d x^{2}}
$$

where $u(x, \tau)$ - the concentration of water vapors, depending on the location and time.

At the boundary between the tank and the tube, i.e. at $x=0$, the vapor concentration in the tank is equal to $u_{o}$. Since the volume of the tank under the condition accepted by us is much larger than the volume of the tube, the value of $u_{o}$ can be considered constant.

We obtain the first boundary condition:

$$
u(0, \tau)=u_{o}
$$

The other end of the tube $(x=L)$ is impervious to vapor molecules.

Mathematically, this condition will be written in the form of:

$$
\left.\frac{d u}{d x}\right|_{x=L}=0
$$

Since $\tau=0$ in the tube no water vapor, we have:

$$
u(x, 0)=0 \text {, }
$$

where $0 \leq x \leq L$

The solution of equation (7) with boundary conditions (8) and (9) and initial condition (10) has the form: 


$$
u(x, \tau)=u_{o}-\frac{4 u_{o}}{\pi} \cdot \sum_{K=0}^{\infty} \frac{1}{2 K+1} \cdot e^{-\frac{(2 K+1)^{2} \pi^{2} D \tau}{4 L^{2}}} \cdot \sin \frac{(2 R+1) \cdot \pi x}{2 L} .
$$

The rest of this series is estimated by the formula [7]:

$$
\left|R_{n}(x, \tau)\right|<\frac{2 u_{o} \cdot e^{-A_{n}^{2}}}{\pi \cdot A_{n}^{2}}
$$

where $A_{n}=\frac{(2 n+1) \pi \sqrt{D \tau}}{2 L}$.

The analysis of the formula (12) shows the expediency in calculating the vapor concentration in equation (11) to reduce the number of series members as time values increase $\tau$. At the maximum $\tau$ value, the series becomes almost zero, and the vapor concentration along the entire length of the capillary is equal to $u_{o}$.

For small values $\tau$, the solution obtained in work [7] is used instead of equation (11):

$$
u(x, \tau)=2 u_{o}\left[1-\Phi\left(\frac{x}{\sqrt{2 D \tau}}\right)+\Phi\left(\frac{x-2 L}{\sqrt{2 D \tau}}\right)\right],
$$

here $\Phi(x)=\frac{1}{\sqrt{2 \pi_{o}}} \int_{o}^{x} e^{-\frac{\alpha^{2}}{2}} \cdot d \alpha$ is the integral of probabilities.

Thus $\tau<\tau_{\mathrm{o}}=\frac{0,2 L^{2}}{D}$ it is recommended to use equation (13), $\tau \geq \tau_{\mathrm{o}}$ the following equation:

$$
u(x, \tau)=u_{o}-\frac{4 u_{o}}{\pi} \cdot\left[e^{-\frac{\pi^{2} D \tau}{4 L^{2}}} \cdot \sin \frac{\pi \cdot x}{2 L}+\frac{1}{3} e^{-\frac{9 \pi^{2} D \tau}{4 L^{2}}} \cdot \sin \frac{3 \pi \cdot x}{2 L}\right] .
$$

Simplify the equations (13) and (14) using the change of variables:

$$
C_{1}=\frac{2 D}{L^{2}}, \quad C_{2}=\frac{x}{L} .
$$

After transformation of equations (13) and (14) we obtain:

for $\tau<\tau_{o}$

$$
u(x, \tau)=2 u_{o}\left[1-\Phi\left(\frac{C^{2}}{\sqrt{C_{1}}}\right)+\Phi\left(\frac{C_{2}-2}{\sqrt{C_{1}}}\right)\right]
$$

for $\tau \geq \tau_{O}$

$$
u(x, \tau)=u_{o}-\frac{4 u_{o}}{\pi} \cdot\left[e^{-\frac{\pi^{2} C_{1}}{8}} \cdot \sin \frac{\pi C_{2}}{2}+\frac{1}{3} \cdot e^{-\frac{9 \pi^{2} C_{1}}{8}} \cdot \sin \frac{3 \pi \cdot C_{2}}{2}\right]
$$

As follows from the analysis of equations (16) and (17), concentration is a function of only three variables $-u_{o}, C_{1}, C_{2}$, and $u_{o}$ is just a multiplier.

Therefore $u(x, \tau)$, the concentration can be expressed by the equation:

$$
u(x, \tau)=u_{o} \cdot f\left(C_{1}, C_{2}\right),
$$

Where $f\left(C_{1}, C_{2}\right)$ is determined by either equation (16) or equation (17). 
From the analysis of the function $f\left(C_{1}, C_{2}\right)$ presented in the form of a table in the work [7], it follows that the average concentration of water vapor in the tube, equal to $u_{o} / 2$, will come after a time $\tau$ :

$$
\tau=0,22 \frac{L^{2}}{D}
$$

and to create a $95 \%$ concentration of water vapor in the tube it will take time:

$$
\tau=1,95 \frac{L^{2}}{D}
$$

In real capillaries (microfields), the intensity of moisture transfer is significantly affected by the small size of their diameters and torsion.

There is a direct proportional relationship between the diffusion coefficient and the mean free path of molecules (formula 2). The average path length of molecules near the capillary walls will be less due to the collision of vapor molecules not only with each other, but also with the walls.

The average radius of capillaries $2,48 \cdot 10^{-6} \mathrm{~m}[4$, about five times the average path length of vapor molecules $\bar{\lambda}=0,906 \cdot 10^{-6} \mathrm{~m}$ (at temperature $t=50^{\circ} \mathrm{C}$ and pressure $\left.P=12,3 \cdot 10^{3} \mathrm{~Pa}\right)$. Therefore, the diffusion coefficient during the movement of vapor molecules across the capillaries will be $20 \%$, from the average free path of molecules, and when moving vapor molecules along the capillaries even less.

The diffusion coefficient is also affected by the torsion of capillaries for pores of medium and large radii, when moving in them, molecules often collide with walls, but due to the small size $\bar{\lambda}$, this effect will be insignificant.

When the longitudinal diffusion coefficient in the middle and wide capillaries is approximately the same as far away from the walls, we determine the time during which the average vapor concentration in the capillary with an average torsion coefficient $\xi=2,0 \mathrm{~m} / \mathrm{m}$ and a shell thickness of $1,5 \mathrm{~mm}$ will be equal to $95 \%$ of the vapor concentration in the tank:

$$
\tau=1,95 \frac{\left(1,5 \cdot 10^{-3} \cdot 2\right)^{2}}{1,87 \cdot 10^{-4}}=9,39 \cdot 10^{-2} \approx 10^{-1} \mathrm{c} .
$$

In microcapillaries $\left(\mathrm{r}<10^{-7} \mathrm{~m}\right)$, the mean free path length of the vapor molecule in the transverse direction is approximately the diameter of the capillary, in which case the microcapillaries are filled within a few seconds.

\section{Summary}

1) When using the pre-vacuuming of the internal micropolities of the grain through its porous shell to reduce the time spent on moistening the grain before grinding should take into account moisture transfer by capillaries (micropolities) grain shell in the gaseous phase (in the form of steam) as a more effective [8].

2) When exposed to steam in a pre-pressurized cavity of the shell of the grain further concentration occurs within a few seconds.

3) One of the variants of the proposed devices for intensification of the process of moisturizing the grain before grinding can be known devices $[9,10]$ used in the processing of hygrothermal pre-vacuumed Shoe parts of their natural leathers. The improvement of these devices, as applied to grain handling industry is a goal for further research. 


\section{References}

1. G.A. Egorov. Technology offlour, groats (1984)

2. I. N. Krasnov, O. F. Cross, I. A. Kravchenko, Method of moistening the grain before grinding: Pat. 2465048 ROS. Federation, publ. (2012)

3. K.A. Adigamov., L.V. Larina. Vacuum and sorption moistening of blank footwear uppers. Monograph (South Russian. State. Tech. Univ. of Illinois, 2000)

4. A.V. Lykov, Heat and mass transfer: Handbook. 2nd ed. Rev. and extra (1978)

5. V.M. Jaworski., A.A. Detla. Handbook of physics (1979)

6. A.V. Lykov, Transport Phenomenon in capillary-porous bodies (1954)

7. G.V. Filki, Methods of calculation of fields of concentration of polluting substances entering the watercourses with sewage: dis. kand. tech. Sciences (1986)

8. L.V. Larina, Methodology of the study and development of processes and equipment for processing natural leather hygrothermal effects on their microstructure under vacuum conditions: abstract. dis. Dr. tech. Sciences (2014)

9. L. V. Larina, B. I. Surmilov, A. Zagoskin. Device for hygrothermal fixing workpieces top shoes: Pat. 2312573 ROS. Federation, publ. (2007)

10. L. V. Larina, V. V. Smirnov, O. B. Tikhonova, E. I. Smirnova Device for molding blanks-top shoes: Pat. 2537160 ROS. Federation, publ. (2014) 